\title{
A população idosa de juiz de fora e os gastos com medicamentos: um estudo transversal
}

\author{
The elderly population of Juiz de Fora and the expenses with medicines: a cross-sectional \\ study
}

La población idosa de Juiz de Fora y los gastos con medicamentos: un estudio transversal

Thaís Fernandes de Oliveira ${ }^{1 *}$, Guillermo Patrício Ortega Jácome ${ }^{1}$, Nathália Barbosa do Espírito Santo Mendes ${ }^{1}$, lanka Cristina Ernesto ${ }^{1}$, Karina Mendes Seghetto ${ }^{1}$, Kaíque Pereira Amorim ${ }^{1}$, Marcela Mattar de Melo Miranda Salim¹, Ana Luiza Marins Franco ${ }^{1}$, Laura Maia Guzowski', Lília Alves dos Santos ${ }^{1}$, Ana Caroline Melo Barros ${ }^{1}$, Isabela Negreiros Ribeiro ${ }^{1}$, Bruna Reboredo Lôbo'.

\section{RESUMO}

Objetivo: Analisar o gasto individual com medicamentos na população acima de 60 anos, demonstrar como os idosos adquirem os medicamentos utilizados e seu perfil epidemiológico. Métodos: Foram selecionados indivíduos com idade mínima de 60 anos, residentes em Juiz de Fora / MG e abordados em locais públicos com alto fluxo populacional, como praças e centros comerciais. Os voluntários realizaram um questionário que forneceu dados com informações sobre a saúde do idoso, utilização do serviço do Sistema Único de Saúde (SUS), o uso de medicações e seu gasto mensal. Resultados: Após a entrevista de 233 indivíduos, observou-se uma predominância do sexo feminino (60,5\%) e a idade média dos participantes foi de 69 anos. Os anti-hipertensivos foram os mais utilizados e, dentre estes, o subgrupo dos Bloqueadores do Receptor de Angiotensina. A faixa etária de 70-79 anos foi a que mais utilizou anti-hipertensivos, antidiabéticos, estatinas e antiagregantes plaquetários. O gasto médio mensal com medicamentos foi $R \$ 201,30$. Conclusão: $A$ hipertensão arterial sistêmica é a doença responsável por maior parte dos medicamentos utilizados e o gasto com medicamentos é proporcional ao envelhecimento, principalmente entre usuários do SUS.

Palavras-chave: Idosos, Saúde do idoso, Gastos, Medicamentos.

\begin{abstract}
Objective: To analyze the individual spending on medicines in the population over 60 years old, to demonstrate the way in which the elderly acquire the medicines used and their epidemiological profile. Methods: We selected individuals with a minimum age of 60 years, living in Juiz de Fora / MG and approached in public places with high population flow, such as squares and shopping centers. The volunteerees perfomed a questionnaire that provided data with information on the health of the elderly, use of the Unified Health System (SUS) service, the use of medications and their monthly expenditure. Results: After interviewing 233 individuals, there was a predominance of females $(60,5 \%)$ and the average age of participants was 69 years. Antihypertensive drugs were the most used and among these, the subgroup of Angiotensin Receptor Blockers. The age group of 70-79 years was the one that most used antihypertensive, antidiabetic, statin and antiplatelet agents. The average monthly expenditure on medicines was $\mathrm{R} \$ 201,30$. Conclusion: That systemic arterial hypertension is the disease responsible for most of the drugs used and drug spending is proportional to aging, especially among elderly SUS users.
\end{abstract}

Key words: Elderly, Elderly health, Expenses, medicines.

${ }^{1}$ Centro Universitário Presidente Antônio Carlos - UNIPAC, Juiz de Fora (Minas Gerais).

*E-mail: athaisfernandesdeoliveira@gmail.com

SUBMETIDO EM: 11/2019

ACEITO EM: 11/2019

PUBLICADO EM: 11/2019 


\section{RESUMEN}

Objetivo: Analizar el gasto individual en medicamentos en la población mayor de 60 años, demostrar como los ancianos adquieren los medicamentos utilizados y su perfil epidemiológico. Métodos: Seleccionamos individuos con edad mínima de 60 años, que viven en Juiz de Fora / MG y fuemos a lugares públicos con alto flujo de población, como plazas y centros comerciales. Los voluntarios realizaron un cuestionario que proporcionaba datos con información sobre la salud de los ancianos, el uso del servicio del Sistema Unificado de Salud (SUS), el uso de medicamentos y su gasto mensual. Resultados: Después de entrevistar a 233 individuos, predominó el sexo femenino $(60,5 \%)$ y la edad promedio de los participantes fue de 69 años. Los fármacos antihipertensivos fueron los más utilizados y entre estos, el subgrupo de Bloqueadores del Receptor de Angiotensina. El grupo de edad de 70-79 años fue el que más utilizó agentes antihipertensivos, antidiabéticos, estatinas y antiplaquetarios. El gasto mensual promedio en medicamentos fue $R \$ 201,30$. Conclusión: La hipertensión arterial sistémica es la enfermedad responsable de la mayoría de los medicamentos utilizados y el gasto de medicamentos es proporcional al envejecimiento, especialmente entre los usuarios mayores de SUS.

Palabras-clave: Ancianos, Salud del anciano, Gastos, Medicamentos.

\section{INTRODUÇÃO}

A partir dos anos 1970, iniciou-se, no Brasil, um processo denominado de transição demográfica, com crescente urbanização e redução nas taxas de natalidade e mortalidade da população (LEONE ET, et al., 2010). Como consequência, o número de pessoas com mais de 60 anos é cada vez maior (VASCONCELOS AMN e GOMES MMF, 2012).

Nos últimos anos, a população idosa brasileira apresentou um incremento quase duas vezes superior ao da população geral, sendo o segmento populacional que mais tem aumentado (SILVA AL, et al., 2012). Isto se deve ao fato de que a expectativa de vida ao nascer aumentou consideravelmente ao longo da história, em grande parte, devido à redução da mortalidade infantil, consequência da diminuição da mortalidade por doenças infecciosas. Além disso, nas últimas três décadas, a mortalidade dos idosos também tem diminuído substancialmente (FAUSTINO CG, 2017). Estima-se que os idosos brasileiros venham a constituir $14 \%$ da população total, ocupando, assim, o sexto lugar na classificação mundial (INOUYE K, et al., 2008).

Sendo assim, os idosos têm despertado uma demanda de atenção significante, posto que o envelhecimento populacional é uma característica etária que avalia a assistência à saúde e o desenvolvimento de uma nação. $O$ crescimento da população acima de 60 anos precisa acontecer juntamente com uma maior qualidade de vida, pois, com o avançar da idade, os números relacionados às Doenças Crônicas Não Transmissíveis (DCNT) aumentam, os quais representam o maior potencial de morbimortalidade no Brasil (DUNCAN BB, et al., 2012).

O envelhecimento da população requer atenção por parte do governo em seus setores de saúde e previdência social, uma vez que envelhecimento e adoecimento não são sinônimos. Excluindo-se hipóteses de comorbidades, o envelhecimento é um indicador de qualidade de vida (MIRANDA GMD, et al., 2016). Paralelamente, como tem sido observado um aumento gradativo da longevidade da população, resultante da diminuição das taxas de mortalidade nas últimas décadas, surgem novas demandas sociais, porquanto há maior prevalência de doenças crônico-degenerativas nesta faixa etária, tais como: doenças cardiovasculares, respiratórias, neoplasias, diabetes mellitus (DM), distúrbios no trato gastrintestinal (TGI), perturbações psicológicas, entre outras (NETO JAC, et al., 2012). Logo, o fator prevenção impõe sua importância quando se percebe que a ausência de lazer, educação, alimentação e outros direitos do cidadão colaboram com o grau de saúde do idoso. As necessidades socioeconômicas, quando impedem uma boa vivência do envelhecimento, determinam uma situação patológica do envelhecer (VILELA ABA, et al., 2006).

Em acréscimo à prevalência das doenças crônicas, a população idosa também é caracterizada pela alta utilização de medicamentos (LIMA MG, et al., 2007). A maior prevalência de comorbidades faz dos idosos 
grandes consumidores de serviços de saúde e, possivelmente, o grupo mais medicalizado da sociedade (SANTOS TRA, et al, 2013). Uma parcela considerável da população idosa apresenta, concomitantemente, inúmeras doenças, o que já determina o uso regular de três ou mais fármacos. Entretanto, a senilidade já é um fator que demanda uso de medicações, pois provoca alterações na composição corporal e nas funções renal e hepática provocadas pelo envelhecimento humano natural (GORZONI ML, et al, 2012).

A alta demanda de medicamentos utilizados pela população senil traz consigo riscos. Além da prescrição inadequada de fármacos, outro problema encontrado é a falta de adesão medicamentosa por parte dos idosos. Entre os fatores que influenciam essa condição estão: a ausência de vínculo empregatício antes da aposentadoria, o declínio cognitivo e os hábitos alimentares inadequados (ARRUDA DCJ, et al., 2015). Os cuidadores e membros familiares exercem uma possível influência positiva na adesão do tratamento medicamentoso, principalmente nos idosos com déficit cognitivo (AIOLFI CR, et al., 2015).

Diante da necessidade farmacológica inevitável, o Programa Farmácia Popular do Brasil foi instituído, em 2004, no contexto do Sistema Único de Saúde (SUS). A referida iniciativa do Governo Federal objetiva maximizar o alcance aos medicamentos, sendo direcionada à uma assistência populacional equitativa e integral. Esse plano governamental visa impedir a desistência de terapêuticas, sobretudo na parcela populacional de baixa renda que faz uso de serviços particulares de saúde, mas que, mesmo assim, encontra obstáculos em obter os medicamentos prescritos em drogarias comerciais. Ainda que esse programa tenha direcionamento para todos os indivíduos, independente da faixa de idade a que pertencem, é particularmente relevante para idosos, cujas necessidades em saúde são mais frequentes, o que gera um maior uso dos serviços de saúde e fármacos. Ademais, tal programa configura-se como uma medida extremamente relevante no controle e prevenção de doenças crônicas, ampliando o alcance da população e minimizando as consequências do gasto com medicamentos no planejamento financeiro familiar (MIRANDA VIA, et al., 2016).

Muitas doenças crônicas têm como um importante fator de risco a idade. Por isso, os gastos com medicamentos são uma realidade nessa faixa etária. Como a maioria da população idosa que apresenta alguma comorbidade usa parte do salário da aposentadoria para comprar medicamentos, é importante saber a quantidade do gasto com eles a fim de analisar o impacto dessa despesa no custo de vida (NETO JAC, et al., 2012).

Além disso, é relevante definir o perfil da população acima de 60 anos de Juiz de Fora, quais fármacos são usados e identificar as enfermidades que contam com um maior apoio governamental no que diz respeito ao seu tratamento. Dado que uma porcentagem da população compromete grande parte do orçamento familiar na compra de remédios, iniciativas que promovam a disponibilidade de drogas gratuitas ou com descontos são essenciais para assegurar a preservação da saúde do idoso, pois um percentual deles abandona o tratamento por falta de condições financeiras (MIRANDA VIA, et al., 2016). O objetivo do presente estudo foi analisar o gasto individual da cidade de Juiz de Fora, Minas Gerais (MG) a respeito dos medicamentos na população acima de 60 anos, bem como demonstrar a forma ao qual os idosos entrevistados adquirem os medicamentos utilizados e seu perfil epidemiológico.

\section{MÉTODOS}

Trata-se de um estudo um estudo observacional do tipo transversal, de base populacional, com idosos da cidade de Juiz de Fora, Minas Gerais. Os idosos foram abordados no período diurno em locais públicos, como o Terminal Rodoviário Miguel Mansur, na zona Norte de Juiz de Fora, e praças com acesso a assentos, como: o Parque Halfeld, na zona central da cidade; e a Praça Santa Luzia, na Zona Sul, resultando em uma melhor comodidade. Eles foram convidados a participar do referido projeto. Foram incluídos na pesquisa as pessoas maiores de sessenta anos de idade que aceitaram participar da entrevista.

Foi aplicado um questionário com informações socioculturais (sexo, idade, estado civil, moradia, profissão, escolaridade e nível econômico) e relacionadas à saúde do idoso, confeccionado especificamente para essa pesquisa. As questões abordam a frequência de internações nos últimos doze meses, quantidade de consultas médicas no período de um ano, dificuldades relacionados a marcações de consultas, utilização do 
serviço do Sistema Único de Saúde (SUS), presença de comorbidades (hipertensão arterial sistêmica e diabetes mellitus), gasto mensal médio com medicamentos nos últimos três meses, problemas encontrados ao necessitar do medicamento, quais medicamentos utilizados, o porquê do uso, quando iniciou, dose e quantidade, onde o medicamento foi adquirido e quem indicou o medicamento.

Os dados foram obtidos durante o período de dezembro de 2018 a março de 2019 e armazenados no programa Access 2013, Microsoft Corporation ${ }^{\circledR}$ USA. Para a análise estatística, foi utilizado o programa Statistical Package for the Social Sciences 21.0, da empresa International Business Machine (IBM ${ }^{\circledR} S P S S$ Statistics 21.0). Medidas de posição e tendência central foram utilizadas para a descrição de variáveis contínuas e proporções para as variáveis categóricas estudadas. $\mathrm{Na}$ análise bivariada foram verificadas as diferenças entre variáveis contínuas através do teste $T$ de igualdade de duas amostras independentes. Em variáveis categóricas para verificar diferenças entre duas amostras independentes foi utilizado o teste de quiquadrado.

$\mathrm{Na}$ análise do $p$-valor e os intervalos de confiança o valor crítico foi definido em $95 \%$. Os dados foram agrupados e apresentados em tabelas e gráficos. Esta coleta de dados foi realizada de forma anônima e após aceitação da participação no estudo, mediante leitura e assinatura do Termo de Consentimento Livre e Esclarecido (TCLE).

Foi solicitado aos idosos participantes da pesquisa sinceridade ao responder ao questionário. Foi ressaltado pelos pesquisadores que os dados são usados para fins exclusivamente científicos. As informações individuais são confidenciais sem identificação de pessoas, mantendo-se o sigilo dos dados. $O$ preenchimento do questionário não foi obrigatório, sendo permitida sua devolução em branco. No caso de indivíduos analfabetos, analfabetos funcionais ou com dificuldade de ler e/ou escrever, o preenchimento do questionário foi feito por entrevista guiada pelo pesquisador responsável pela aplicação do respectivo questionário.

A pesquisa se baseou nas diretrizes e normas éticas regulamentadoras de pesquisas envolvendo seres humanos determinadas pela Resolução do Conselho Nacional de Saúde (CNS) n466, de 12 de dezembro de 2012. Considera-se que o trabalho apresentou risco mínimo, o mesmo que toda pessoa está exposta no dia-a-dia. O presente estudo foi aprovado pelo Comitê de Ética em Pesquisa (CEP) com seres humanos da Santa Casa de Misericórdia de Juiz de Fora, protocolo número 66615017.2.0000.5139.

\section{RESULTADOS}

Considerando que o presente estudo abrangeu uma população que informou, randomicamente, ter concluído ensino fundamental, ensino médio e ensino superior (escolaridades baixa, média e alta, respectivamente), a maioria dos idosos era aposentada, casada e relatou ter média escolaridade (Tabela 1).

Tabela 1 - Características sociodemográficas dos idosos com idade $\geq 60$ anos, Juiz de Fora - MG, 2018.

\begin{tabular}{lc}
\hline Variável & $\mathbf{N}(\%)$ \\
\hline Sexo masculino & $92(39,5)$ \\
Sexo feminino & $141(60,5)$ \\
\hline Atividade & \\
Aposentado & $115(49,4)$ \\
Ativo & $59(25,5)$ \\
\hline Escolaridade & \\
Baixa & $44(18,9)$ \\
Média & $54(23,2)$ \\
Alta & $51(21,9)$ \\
\hline Estado civil & \\
Solteiro/divorciado & $56(24,0)$ \\
Casado & $127(54,5)$ \\
\hline
\end{tabular}

Fonte: Oliveira TF, et al., 2019. 
Na população entrevistada $(n=233$ ), observou-se uma predominância do sexo feminino, correspondendo a $60,5 \%$ dos idosos. A idade média dos participantes foi de 69 anos - desvio padrão (DP) de 8,7 anos -, com uma variação de 60 a 93 anos.

A seguir, observa-se que o grupo terapêutico mais utilizado foi de anti-hipertensivos e, dentre estes, destacou-se o subgrupo dos Bloqueadores do Receptor de Angiotensina (BRA), seguido do subgrupo dos diuréticos. Cerca de $19 \%$ dos idosos relataram o uso de antidiabéticos, com predomínio da metformina (88,9\% dos diabéticos) (Tabela 2).

Tabela 2 - Frequência de utilização de medicamentos segundo os grupos terapêuticos, Juiz de Fora - MG, 2018.

\begin{tabular}{cc}
\hline Grupos terapêuticos (n=233) & Frequência (\%) \\
\hline Anti-hipertensivos & 61,4 \\
Estatinas & 23,2 \\
Antidiabéticos & 19,3 \\
Inibidores da bomba de próton & 18,0 \\
Antiagregantes plaquetários & 14,6 \\
\hline Subgrupos terapêuticos & \\
Anti-hipertensivos (n=143) & Frequência (\%) \\
\hline IECA & 24,5 \\
BRA & 59,4 \\
Diurético & 49,7 \\
BCC & 25,2 \\
Betabloqueador & 26,6 \\
\hline Antidiabéticos (n=50) & \\
\hline Metformina & 88,9 \\
Incretinomiméticos & 8,9 \\
Inibidores do SGLT2 & 2,2 \\
Insulina & 13,3 \\
Glitazonas & 4,4 \\
\hline
\end{tabular}

Legenda: Inibidores da Enzima de Conversão da Angiotensina (IECA), Bloqueadores de Receptores de Angiotensina (BRA), Bloqueadores dos Canais de Cálcio (BCC), Inibidores do cotransportador do sódioglicose 2 (SGLT2).

Fonte: Oliveira TF, et al., 2019.

A faixa etária de 70-79 anos foi a que mais utilizou anti-hipertensivos, antidiabéticos, estatinas e antiagregantes plaquetários (Tabela 3 ).

Tabela 3 - Frequência de utilização dos grupos terapêuticos segundo a faixa etária, Juiz de Fora - MG, 2018.

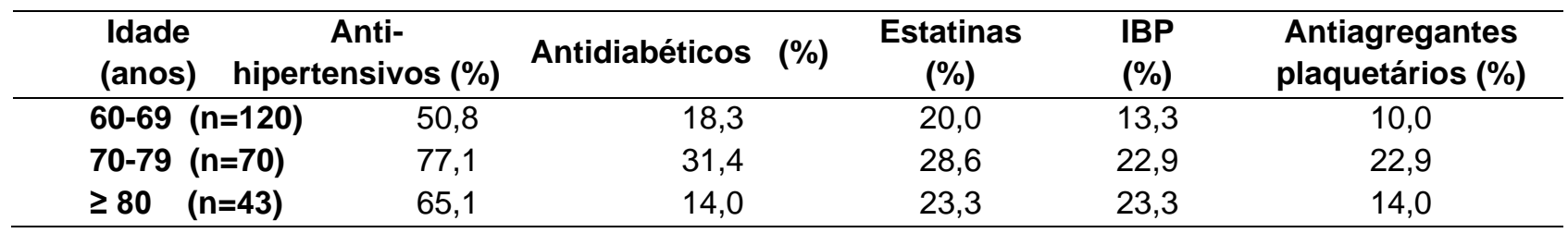

Legenda: Inibidores da Bomba de Prótons (IBP).

Fonte: Oliveira TF, et al., 2019.

O gasto médio mensal com medicamentos foi de $R \$ 201,30$, com o valor mínimo de $R \$ 13,00$, o máximo de $R \$ 1.800,00$ e a mediana de $R \$ 100,00$. Os maiores gastos foram entre os não usuários do SUS e entre os da faixa etária de 70-79 anos (Tabela 4). 
A média de medicamentos utilizados foi 3,5, o mínimo foi 1, o máximo foi 12 e a mediana foi 3.

Tabela 4 - Gasto com medicamentos segundo a faixa etária em idosos. Juiz de Fora, MG, 2018 ( $n$ = 233).

\begin{tabular}{l|lccccc}
\hline \multicolumn{1}{l}{} & & $\begin{array}{c}\text { Gasto médio } \\
\text { mensal (R\$) }\end{array}$ & $\begin{array}{c}\text { Desvio } \\
\text { Padrão }\end{array}$ & $\begin{array}{c}\text { Média de } \\
\text { medicamentos }\end{array}$ & $\begin{array}{c}\text { Desvio } \\
\text { Padrão }\end{array}$ & p-valor \\
\hline \multirow{4}{*}{ Utiliza SUS } & $\mathbf{6 0 - 6 9}$ anos & 134,90 & 263,00 & 3,1 & 2,2 & \\
& $\mathbf{7 0 - 7 9}$ anos & 177,90 & 240,00 & 4,5 & 2,4 & \\
& $\geq \mathbf{8 0}$ anos & 191,30 & 232,20 & 4,4 & 2,7 & 0,526 \\
& Média total & {$[157,50]$} & & {$[3,7]$} & & \\
\hline \multirow{4}{*}{ Não utiliza SUS } & $\mathbf{6 0 - 6 9}$ anos & 170,90 & 210,30 & 2,8 & 2,5 & \\
& $\mathbf{7 0 - 7 9}$ anos & 385,90 & 456,40 & 3,3 & 2,2 & \\
& $\geq 80$ anos & 313,30 & 359,40 & 3,6 & 2,8 & 0,022 \\
& Média total & {$[265,00]$} & & {$[3,1]$} & & \\
\hline
\end{tabular}

Fonte: Oliveira TF, et al., 2019.

Foi observado maior gasto no grupo de mulheres com alta escolaridade e menor gasto no grupo de homens com alta escolaridade. Entretanto, não houve diferença estatisticamente significativa quanto ao gasto mensal médio com medicamentos entre os diferentes níveis de escolaridade (Tabela 5).

Tabela 5 - Comparação entre o nível de escolaridade e o gasto com medicamentos por idosos. Juiz de Fora, MG, 2018.

\begin{tabular}{cccccc}
\hline & \multicolumn{2}{c}{ Sexo feminino } & \multicolumn{3}{c}{ Sexo masculino } \\
\hline Escolaridade & $\begin{array}{c}\text { Gasto médio } \\
\text { mensal }(\mathbf{R} \$)\end{array}$ & $\begin{array}{c}\text { Desvio } \\
\text { padrão }\end{array}$ & $\begin{array}{c}\text { Gasto médio } \\
\text { mensal }(\mathbf{R} \$)\end{array}$ & $\begin{array}{c}\text { Desvio } \\
\text { padrão }\end{array}$ & p-valor \\
\hline Baixa $(\mathbf{n}=66)$ & 184,00 & 218,30 & 224,10 & 362,10 & \\
Média $(\mathbf{n}=\mathbf{1 0 8})$ & 175,20 & 280,90 & 178,20 & 214,10 & \\
Alta $\quad(\mathbf{n = 5 5 )}$ & 292,00 & 418,20 & 206,10 & 238,10 & 0,423 \\
\hline
\end{tabular}

Fonte: Oliveira TF, et al., 2019.

\section{DISCUSSÃO}

Analisando os dados obtidos, constatou-se que a maioria da população entrevistada era pertencente ao sexo feminino $(39,5 \%)$, fato justificado pelo processo de feminização da velhice. A maior longevidade da mulher é consequência da maior expectativa de vida da mesma, que vive, em média, 8 anos a mais que os homens, uma vez que estes morrem precocemente por serem, em 90\% dos casos, vítimas de homicídios e acidentes. Ademais, a população feminina é mais fiel ao seguimento médico de rotina ao longo de suas vidas, sendo alvo mais eficaz na prevenção e controle de doenças crônico-degenerativas (KÜCHEMANN BA, 2012).

Em relação à idade, neste estudo encontrou-se um valor médio de 69 anos (DP de 8,7 anos), uma projeção intensa do processo de transição demográfica que, determinando uma maior expectativa de vida, culmina em uma população de 60 anos ou mais ganhando um espaço cada vez maior em relação à população mais jovem. (VASCONCELOS AMN, GOMES MMF, 2012).

Quanto às comorbidades, a maioria dos idosos analisados $(61,4 \%)$ relatou ser portadora de hipertensão arterial sistêmica (HAS), que é a confirmação de um perfil epidemiológico para tal faixa etária no país pois, entre as doenças crônicas mais frequentes nos maiores de 60 anos, destacam-se a Hipertensão Arterial Sistêmica (HAS) e o Diabetes mellitus (DM) (DUNCAN BB, et al., 2012). Percebe-se, portanto, a necessidade do acompanhamento médico contínuo nessa parcela da população visando diminuir a morbimortalidade, uma vez que a hipertensão é fator de risco para várias outras doenças cardiovasculares.

No que diz respeito às classes farmacológicas mais utilizadas pelos idosos entrevistados, obteve-se os seguintes resultados: primeiramente, os anti-hipertensivos, em destaque BRA $(59,4 \%)$ e diuréticos $(49,7 \%)$; em seguida as estatinas; em terceiro lugar os antidiabéticos; em quarto os inibidores de bomba de próton, e 
por último os antiagregantes plaquetários. Nesse contexto, as informações encontradas traçam um perfil farmacológico comum entre os idosos no Brasil: o grande uso de anti-hipertensivos e estatinas é justificado pela alta prevalência doenças cardiovasculares, sendo estas as líderes das causas de morbimortalidade entre indivíduos com idade acima de 65 anos (NETO JAC, et al., 2012).

Sobre os medicamentos utilizados entre os idosos entrevistados no corrente estudo, a média de fármacos em uso foi 3,5, o mínimo foi 1, o máximo foi 12 e a mediana foi 3 . A análise dos resultados obtidos quanto à quantidade e variedade de medicamentos em uso gera preocupação em relação à polifarmácia na população abordada. Embora os idosos necessitem do uso de fármacos para doenças crônicas e quadros específicos faz-se necessária uma abordagem médica mais criteriosa sobre essa parcela da população, uma vez que a utilização simultânea de várias classes farmacológicas pode propiciar a ocorrência de iatrogenias (BEZERRA TA, et al., 2015).

Em referência aos gastos com medicamentos, foi analisado um gasto mensal médio de $R \$ 201,30$ entre os idosos entrevistados e a mediana, $R \$ 100,00$. Nos resultados obtidos pelo inquérito da população participante do estudo, o valor máximo obtido foi igual a $R \$ 1.800,00$, valor este encontrado entre usuários do setor privado; e o valor mínimo obtido foi igual a $\mathrm{R} \$ 13,00$, achado entre usuários do SUS. Em pesquisa realizada na cidade de Belo Horizonte, o gasto mensal médio individual privado foi $\mathrm{R} \$ 122,97$ e a mediana $\mathrm{R} \$ 69,91$. Dos gastos privados mensais, $53,6 \%$ foram com medicamentos de referência, $41,7 \%$ com similares e apenas $4,7 \%$ com genéricos. Existe também discordância quanto ao gasto mensal no setor privado, que em Juiz de Fora foi maior que o do estudo em questão, mas os dados não possuem valor estatístico relevante (FAUSTINO CG, 2017).

No presente estudo, percebeu-se que o maior gasto com medicamentos estava associado aos idosos do sexo feminino e com alta escolaridade, enquanto as menores despesas mensais pertenciam ao grupo de homens com alta escolaridade. A literatura relaciona o consumo farmacológico e o nível de conhecimento dos indivíduos, sendo a escolaridade um fator correlacionado ao maior número de consultas médicas. Dessa forma, pode-se inferir que indivíduos mais instruídos constatam com mais eficiência suas necessidades, recorrem mais frequentemente ao apoio profissional adequado e têm mais liberdade para acessar o sistema de saúde, assim como ao consumo e eventual gasto com medicamentos (PANIZ VMV, et al., 2008).

Os maiores gastos financeiros mensais foram entre os não usuários do SUS, provavelmente por serem idosos de uma classe econômica mais favorável. De acordo com a literatura, o gasto com medicamento varia de acordo com a classe econômica. Essa diferença tem relação com o fato de que a classe $A$, dotada de maior renda per capita, adquire seus medicamentos principalmente de forma privada, enquanto as outras classes acessam os medicamentos gratuitos a partir do sistema de saúde público. Além disso, a classe A utiliza preferencialmente medicamentos recentemente lançados no mercado, enquanto que, na classe média, isso é influenciado por oscilações nos preços; na classe $\mathrm{E}$, há uma dependência dos medicamentos adotados pela gestão pública, sendo que o aumento das despesas médicas é diretamente proporcional ao envelhecimento (COLET CF, et al., 2016).

Através do corrente estudo, observou-se que os maiores gastos foram entre os idosos da faixa etária de 70-79 anos e o aumento do gasto mensal foi diretamente proporcional ao envelhecimento em pacientes que utilizam o SUS. Enquanto, ao avaliar os resultados dos pacientes não usuários do SUS, percebemos que na faixa etária entre 70 a 79 anos houve um gasto mensal maior do que a faixa etária de maiores de 80 anos.

Esses dados controversos vão contra ao aumento dos gastos correlacionado com a polipatologia devido ao envelhecimento e apresentam um valor de $p$ igual a 0,022, demonstrando relevância estatística. As doenças não-transmissíveis impõem uma carga financeira a muitas famílias, particularmente nas que residem em países mais pobres. Uma revisão sistemática realizada na cidade de Ribeirão Preto teve por objetivo investigar o ônus financeiro das doenças não transmissíveis em países de média e baixa renda. Apesar da heterogeneidade dos artigos selecionados, quanto aos objetivos e métodos utilizados verificou-se que o custo de se obter tratamento para doenças não transmissíveis está se tornando uma das causas de empobrecimento e catástrofe financeira nas famílias desses países (BALDONI AO e PEREIRA LRL, 2011). 
As ferramentas farmacoepidemiológicas são utilizadas para auxiliar no uso racional dos recursos destinados à aquisição de importantes instrumentos para promover a saúde, sendo assim de fundamental importância para o cumprimento da legislação Brasileira, que assegura a assistência terapêutica integral ao paciente, pois constando no Estatuto do Idoso é incumbência ao Poder Público fornecer aos idosos, gratuitamente, medicamentos, especialmente os de uso continuado (BALDONI AO e PEREIRA LRL, 2011).

A partir desse direito constitucional acima referenciado foi instituído o Programa Farmácia Popular do Brasil, cujo conhecimento atinge $80 \%$ dos idosos, segundo um estudo realizado na cidade de Pelotas - RS. As dificuldades encontradas para o uso deste plano governamental são a ausência de receita médica, falta do medicamento e o mesmo não ser incluído no referido programa, apontando obstáculos no alcance à consulta, além do planejamento deficiente da oferta e fornecimento dos medicamentos.

Sendo assim, faz-se necessário constatar se as medicações integrantes do programa abrangem consistentemente as carências terapêuticas da população, ainda que o número de fármacos ofertados pelo plano seja vasto, contando com a maior parte dos medicamentos para doenças crônicas, e a relação de medicamentos seja reavaliada regulamente. Paralelamente, para fármacos não integrantes do plano em questão, há análogos inclusos, mostrando que os médicos desconhecem os medicamentos participantes ou casualmente não valorizam tal recurso para o controle na redução de gastos do paciente (MIRANDA VIA, et al., 2016).

\section{CONCLUSÃO}

Os resultados do presente estudo mostraram que a hipertensão arterial sistêmica é a doença responsável por maior parte dos medicamentos utilizados por idosos, destacando a importância da adoção de estratégias de prevenção de doenças crônicas, que afetam tanto a saúde, quanto a vida financeira do indivíduo. O perfil encontrado que aponta para uma maior frequência ao alto consumo e gastos com medicamentoso foi: ser idoso do sexo feminino e de alta escolaridade. Tal fato este que alerta sobre a necessidade de uma melhor instrução médico-farmacêutica do público em questão quanto à real necessidade do alto uso farmacológico. Foi observado que há uma tendência ao aumento do gasto com medicamentos de acordo com o envelhecimento, principalmente entre idosos usuários do SUS, reforçando a importância de programas do governo, como o da Farmácia Popular do Brasil, que facilita o acesso aos medicamentos por esse grupo. A promoção do uso racional de medicamentos e a disponibilidade de genéricos também contribui para diminuir os gastos com medicamentos por essa população. Ademais, os dados apresentados podem cooperar com os gestores de saúde no planejamento de ações que visam melhorar o acesso aos medicamentos essenciais e promover conscientização quanto ao uso destes, reduzindo a polifarmácia e seus riscos.

\section{REFERÊNCIAS}

1. AIOLFI CR, et al. Adesão ao uso de medicamentos entre idosos hipertensos. Revista Brasileira de Geriatria e Gerontologia, 2015; 18(2): 397-404.

2. ARRUDA DCJ, et al. Fatores associados a não adesão medicamentosa entre idosos de um ambulatório filantrópico do Espírito Santo. Revista Brasileira de Geriatria e Gerontologia, 2015; 18(2): 327-337.

3. BALDONI AO, PEREIRA LRL. O impacto do envelhecimento populacional brasileiro para o sistema de saúde sob a óptica da farmacoepidemiologia: uma revisão narrativa. Revista de Ciências Farmacêuticas Básica e Aplicada, 2011; 32(3): 313-321.

4. BEZERRA TA, et al. Caracterização do uso de medicamentos entre idosos atendidos em uma Unidade Básica de Saúde da Família. Revista Cogitare Enfermagem, 2016; 21(1): 01-11.

5. COLET CF, et al. Perfil de gastos com medicamentos entre idosos em diferentes grupos socioeconômicos. Revista Brasileira de Geriatria e Gerontologia, 2016; 19(4): 591-601.

6. DUNCAN BB, et al. Doenças Crônicas Não Transmissíveis no Brasil: prioridade para enfrentamento e investigação. Revista de Saúde Pública 2012; 46(Supl):126-34.

7. FAUSTINO CG. Gasto em medicamentos por famílias com idosos no Brasil. Dissertação (Doutorado em Medicina Preventiva) - Faculdade de Medicina. Universidade de São Paulo, São Paulo, 2017; 140p.

8. GORZONI ML, et al. Medicamentos potencialmente inapropriados para idosos. Revista da Associação Médica Brasileira, 2012; 58(4): 442-446. 
9. INOUYE K, et al. Octogenários e cuidadores: perfil sociodemográfico e correlação da variável qualidade de vida. Texto e contexto Enfermagem, 2008; 17 (2): 350-357.

10. KÜCHEMANN BA. Envelhecimento populacional, cuidado e cidadania: velhos dilemas e novos desafios. Sociedade e Estado, 2012; 27(1): 165-80.

11. LIMA MG, et al. Composição dos gastos privados com medicamentos utilizados por aposentados e pensionistas com idade igual ou superior a 60 anos em Belo Horizonte, Minas Gerais. Cadernos de Saúde Pública, 2007; 23(6): 14231430.

12. LEONE ET, et al. Mudanças na composição das famílias e impactos sobre a redução da pobreza no Brasil. Economia e Sociedade, 2010; 19(1): 59-77.

13. MIRANDA GMD, et al. O envelhecimento populacional brasileiro: desafios e consequências sociais atuais e futuras. Revista Brasileira de Geriatria e Gerontologia, 2016; 19(3):507-519.

14. MIRANDA VIA, et al. Utilização do Programa Farmácia Popular do Brasil por idosos. Revista de Saúde Pública, 2016; $50: 13$.

15. NETO JAC, et al. Uso de medicamentos por idosos de Juiz de Fora: um olhar sobre a polifarmácia. HU Revista, 2012; 37(3):305-313.

16. PANIZ VMV, et al. Acesso a medicamentos de uso contínuo em adultos e idosos nas regiões Sul e Nordeste do Brasil. Cadernos de Saúde Pública, 2008; 24 (2): 267-280.

17. SANTOS TRA, et al. Consumo de medicamentos por idosos, Goiânia, Brasil. Revista de Saúde Pública, 2013; 47(1):94-103.

18. SILVA AL, et al. Utilização de medicamentos por idosos brasileiros, de acordo com a faixa etária: um inquérito postal. Cadernos de Saúde Pública, 2012; 28(6):1033-45.

19. VASCONCELOS AMN, GOMES MMF. Transição demográfica: a experiência brasileira. Epidemiologia e Serviços de Saúde, 2012; 21(4): 539-548.

20. VILELA ABA, et al. Envelhecimento bem-sucedido: representação de idosos. Revista Saúde.com, 2006; 2(2):101114. 